\title{
SOLVABILITY OF CAUCHY PROBLEM FOR A SYSTEM OF FIRST ORDER QUASILINEAR EQUATIONS WITH RIGHT-HAND SIDES $f_{1}=a_{2} u(t, x)+b_{2}(t) v(t, x), \quad f_{2}=g_{2} v(t, x)$
}

\section{M.V. DONTSOVA}

\begin{abstract}
We consider a Cauchy problem for a system of two first order quasilinear differential equations with right-hand sides $f_{1}=a_{2} u(t, x)+b_{2}(t) v(t, x), f_{2}=g_{2} v(t, x)$. We study the solvability of the Cauchy problem on the base of an additional argument method. We obtain the sufficient conditions for the existence and uniqueness of a local solution to the Cauchy problem in terms of the original coordinates coordinates for a system of two first order quasilinear differential equations with right-hand sides $f_{1}=a_{2} u(t, x)+b_{2}(t) v(t, x)$, $f_{2}=g_{2} v(t, x)$, under which the solution has the same smoothness in $x$ as the initial functions in the Cauchy problem does. A theorem on the local existence and uniqueness of a solution to the Cauchy problem is formulated and proved.

The theorem on the local existence and uniqueness of a solution to the Cauchy problem for a system of two first order quasilinear differential equations with right-hand sides $f_{1}=$ $a_{2} u(t, x)+b_{2}(t) v(t, x), f_{2}=g_{2} v(t, x)$ is proved by the additional argument method. We obtain the sufficient conditions of the existence and uniqueness of a nonlocal solution to the Cauchy problem in terms of the initial coordinates for a system of two first order quasilinear differential equations with right-hand sides $f_{1}=a_{2} u(t, x)+b_{2}(t) v(t, x), f_{2}=g_{2} v(t, x)$. A theorem on the nonlocal existence and uniqueness of the solution of the Cauchy problem is formulated and proved. The proof of the nonlocal solvability of the Cauchy problem for a system of two quasilinear first order partial differential equations with right-hand sides $f_{1}=a_{2} u(t, x)+b_{2}(t) v(t, x), \quad f_{2}=g_{2} v(t, x)$ is based on global estimates.
\end{abstract}

Keywords: first order partial differential equations, Cauchy problem, additional argument method, global estimates.

Mathematics Subject Classification: 35F50, 35F55, 35A01, 35A02, 35A05

\section{INTRODUCTION}

In work [1], by means of an additional argument method, there were found the conditions of nonlocal solvability of the Cauchy problem for the system of form:

$$
\left\{\begin{array}{l}
\partial_{t} u(t, x)+(a u(t, x)+b v(t, x)) \partial_{x} u(t, x)=0, \\
\partial_{t} v(t, x)+(c u(t, x)+g v(t, x)) \partial_{x} v(t, x)=0
\end{array}\right.
$$

where $u(t, x), v(t, x)$ are unknown functions, $a, c, b, g$ are known positive constants, $(t, x) \in \Omega_{T}$, where

$$
\Omega_{T}=\{(t, x) \mid 0 \leqslant t \leqslant T, x \in(-\infty,+\infty), T>0\}
$$

subject to the initial conditions:

$$
u(0, x)=\varphi_{1}(x), \quad v(0, x)=\varphi_{2}(x),
$$

M.V. Dontsova, Solvability of Cauchy Problem for a System of First order Quasilinear EQUATIONS WITH RIGHT-HAND SIDES $f_{1}=a_{2} u(t, x)+b_{2}(t) v(t, x), f_{2}=g_{2} v(t, x)$.

(C)Dontsova M.V. 2019.

The reported study was funded by RFBR according to the research project no. 18-31-00125 mol_a.

Submitted May 10, 2018. 
and $\varphi_{1}(x), \varphi_{2}(x)$ are given functions.

In work [2], again by means of the additional argument method there were established the conditions for nonlocal solvability of the Cauchy problem for the system of the form:

$$
\left\{\begin{array}{l}
\partial_{t} u(t, x)+\left(a_{1} u(t, x)+b_{1} v(t, x)\right) \partial_{x} u(t, x)=a_{2} u(t, x)+b_{2} v(t, x), \\
\partial_{t} v(t, x)+\left(c_{1} u(t, x)+g_{1} v(t, x)\right) \partial_{x} v(t, x)=g_{2} v(t, x),
\end{array}\right.
$$

where $u(t, x), v(t, x)$ are unknown functions, $a_{1}, c_{1}, g_{1}, b_{i}, i=1,2$, are given positive constants, $a_{2}, g_{2}$ are given constants, $(t, x) \in \Omega_{T}$, subject to initial conditions (2).

We consider the system of form:

$$
\left\{\begin{array}{l}
\partial_{t} u(t, x)+\left(a_{1}(t) u(t, x)+b_{1}(t) v(t, x)\right) \partial_{x} u(t, x)=a_{2} u(t, x)+b_{2}(t) v(t, x), \\
\partial_{t} v(t, x)+\left(c_{1}(t) u(t, x)+g_{1}(t) v(t, x)\right) \partial_{x} v(t, x)=g_{2} v(t, x)
\end{array}\right.
$$

where $u(t, x), v(t, x)$ are unknown functions, $a_{2}, g_{2}$ are given constants, $a_{1}(t), b_{1}(t), b_{2}(t), c_{1}(t)$, $g_{1}(t)$ are given functions. We suppose that $a_{1}(t)>0, b_{1}(t)>0, b_{2}(t)>0, c_{1}(t)>0, g_{1}(t)>0$, $t \in[0, T]$.

Various methods were employed for studying systems of first order quasilinear equations. In [3], the solvability of a system of first order quasilinear equations was analysed on the base of a classical method of characteristics.

In the framework of the classical method of characteristics, the study is reduced to a nonlinear system of integral equations involving a superposition of unknown functions. Once the solution is found in terms of characteristic variables, to find a solution to the original problem, one has to pass from the characteristic variables to $(t, x)$. In many cases, the latter problem turns out to be so complicated that it is not solved and instead, the possibility of the inverse variables transform is introduced as a condition [3].

In work [4], by means of the additional argument method there were found local solvability conditions of Cauchy problem in terms of original coordinates for a system of two quasilinear equations, under which the solution possesses a lower smoothness than the initial functions $\varphi_{1}(x), \varphi_{2}(x)$, the boundaries of the solvability interval were given.

In the present work, we study system (4) on $\Omega_{T}$ subject to initial condition (2) and we establish sufficient conditions of existence and uniqueness of local solution to this Cauchy problem in original coordinates ensuring the same smoothness in $x$ for the solution as of initial functions. We also provide sufficient conditions for existence and uniqueness of nonlocal Cauchy problem in original coordinates; namely, for a given finite segment $t \in[0, T]$. The results are formulated as corresponding theorems on local and nonlocal unique solvability of the Cauchy problem.

According the additional argument method, for problem (4), (2) we write an extended characteristic system [2], [5]-9]:

$$
\begin{aligned}
& \frac{d \eta_{1}(s, t, x)}{d s}=a_{1}(s) u\left(s, \eta_{1}(s, t, x)\right)+b_{1}(s) v\left(s, \eta_{1}(s, t, x)\right) \\
& \frac{d \eta_{2}(s, t, x)}{d s}=c_{1}(s) u\left(s, \eta_{2}(s, t, x)\right)+g_{1}(s) v\left(s, \eta_{2}(s, t, x)\right) \\
& \frac{d u\left(s, \eta_{1}(s, t, x)\right)}{d s}=a_{2} u\left(s, \eta_{1}(s, t, x)\right)+b_{2}(s) v\left(s, \eta_{1}(s, t, x)\right), \\
& \frac{d v\left(s, \eta_{2}(s, t, x)\right)}{d s}=g_{2} v\left(s, \eta_{2}(s, t, x)\right) \\
& \eta_{1}(t, t, x)=x, \quad \eta_{2}(t, t, x)=x, \\
& u\left(0, \eta_{1}(0, t, x)\right)=\varphi_{1}\left(\eta_{1}(0, t, x)\right), \quad v\left(0, \eta_{2}(0, t, x)\right)=\varphi_{2}\left(\eta_{2}(0, t, x)\right) .
\end{aligned}
$$


We introduce new unknown functions:

$$
\begin{array}{ll}
w_{1}(s, t, x)=u\left(s, \eta_{1}(s, t, x)\right), & w_{2}(s, t, x)=v\left(s, \eta_{2}(s, t, x)\right), \\
w_{3}(s, t, x)=v\left(s, \eta_{1}(s, t, x)\right), & w_{4}(s, t, x)=u\left(s, \eta_{2}(s, t, x)\right) .
\end{array}
$$

Then the characteristic system becomes

$$
\begin{array}{ll}
\frac{d \eta_{1}(s, t, x)}{d s}=a_{1}(s) w_{1}(s, t, x)+b_{1}(s) w_{3}(s, t, x), \\
\frac{d \eta_{2}(s, t, x)}{d s}=c_{1}(s) w_{4}(s, t, x)+g_{1}(s) w_{2}(s, t, x), \\
\frac{d w_{1}(s, t, x)}{d s}=a_{2} w_{1}(s, t, x)+b_{2}(s) w_{3}(s, t, x), \\
\frac{d w_{2}(s, t, x)}{d s}=g_{2} w_{2}(s, t, x), & \\
w_{3}(s, t, x)=w_{2}\left(s, s, \eta_{1}\right), & w_{4}(s, t, x)=w_{1}\left(s, s, \eta_{2}\right), \\
\eta_{1}(t, t, x)=x, & \eta_{2}(t, t, x)=x, \\
w_{1}(0, t, x)=\varphi_{1}\left(\eta_{1}(0, t, x)\right), & w_{2}(0, t, x)=\varphi_{2}\left(\eta_{2}(0, t, x)\right) .
\end{array}
$$

The unknown functions $\eta_{i}, w_{j}, i=1,2, j=\overline{1,4}$, depend not only on $t$ and $x$, but also on an additional argument $s$. Integrating equations (11)-(14) with respect to the argument $s$ and taking into consideration conditions (15)-(17), we obtain an equivalent system of integral equations:

$$
\begin{aligned}
& \eta_{1}(s, t, x)=x-\int_{s}^{t}\left(a_{1}(\tau) w_{1}+b_{1}(\tau) w_{3}\right) d \tau, \\
& \eta_{2}(s, t, x)=x-\int_{s}^{t}\left(c_{1}(\tau) w_{4}+g_{1}(\tau) w_{2}\right) d \tau, \\
& w_{1}(s, t, x)=\varphi_{1}\left(\eta_{1}(0, t, x)\right)+\int_{0}^{s}\left(a_{2} w_{1}+b_{2}(\tau) w_{3}\right) d \tau, \\
& w_{2}(s, t, x)=\varphi_{2}\left(\eta_{2}(0, t, x)\right)+\int_{0}^{s} g_{2} w_{2} d \tau, \\
& w_{3}(s, t, x)=w_{2}\left(s, s, \eta_{1}\right), \\
& w_{4}(s, t, x)=w_{1}\left(s, s, \eta_{2}\right) .
\end{aligned}
$$

We substitute (18), (19) into (20)-23) and we obtain the following system:

$$
\begin{aligned}
& w_{1}(s, t, x)=\varphi_{1}\left(x-\int_{0}^{t}\left(a_{1}(\tau) w_{1}+b_{1}(\tau) w_{3}\right) d \tau\right)+\int_{0}^{s}\left(a_{2} w_{1}(\tau, t, x)+b_{2}(\tau) w_{3}(\tau, t, x)\right) d \tau, \\
& w_{2}(s, t, x)=\varphi_{2}\left(x-\int_{0}^{t}\left(c_{1}(\tau) w_{4}(\tau, t, x)+g_{1}(\tau) w_{2}(\tau, t, x)\right) d \tau\right)+\int_{0}^{s} g_{2} w_{2}(\tau, t, x) d \tau, \\
& w_{3}(s, t, x)=w_{2}\left(s, s, x-\int_{s}^{t}\left(a_{1}(\tau) w_{1}+b_{1}(\tau) w_{3}\right) d \tau\right),
\end{aligned}
$$




$$
w_{4}(s, t, x)=w_{1}\left(s, s, x-\int_{s}^{t}\left(c_{1}(\tau) w_{4}+g_{1}(\tau) w_{2}\right) d \tau\right) .
$$

In what follows, we shall write that constants $K_{0}, K_{1}, K_{2}, \ldots$ are determined by initial data if these constants are determined via known characteristics of the problem, the norms and extrema of known functions by means of closed algebraic, differential or integral expressions, that is, in the framework of the original problem, they can expressed as a certain number.

The following lemma holds true.

Lemma 1. Assume that the functions $w_{1}(s, t, x), w_{2}(s, t, x)$ satisfy system of integral equations (24)-(27), are continuously differentiable and bounded together with its first derivatives. Then the functions $u(t, x)=w_{1}(t, t, x), v(t, x)=w_{2}(t, t, x)$ solve problem (4), (2) on $\Omega_{T_{0}}$, $T_{0} \leqslant T$, where $T_{0}$ is a constant determined by initial data.

Lemma 1 is a base in the additional argument method. Lemma 1 can be proved in the same way as in works [2], 4], [5], [7]-[9].

\section{EXISTENCE OF LOCAL SOLUTIUON}

To prove the existence of solution to problem (4), (2) in the class of bounded functions, we shall employ the system of integral equations $240-(27)$.

We denote

$$
\begin{aligned}
& \Gamma_{T}=\{(s, t, x) \mid 0 \leqslant s \leqslant t \leqslant T, x \in(-\infty,+\infty), T>0\}, \\
& C_{\varphi}=\max \left\{\sup _{R}\left|\varphi_{i}^{(l)}\right| \mid i=1,2, l=\overline{0,2}\right\}, \\
& l=\max \left\{\sup _{[0, T]} a_{1}(t), \sup _{[0, T]} b_{1}(t), \sup _{[0, T]} b_{2}(t), \sup _{[0, T]} c_{1}(t), \sup _{[0, T]} g_{1}(t),\left|a_{2}\right|,\left|g_{2}\right|\right\}, \\
& \|U\|=\sup _{\Gamma_{T}}|U(s, t, x)|,\|f\|=\sup _{\Omega_{T}}|f(t, x)| .
\end{aligned}
$$

The symbol $\bar{C}^{1,2,2}\left(\Omega_{T}\right)$ stand for the space of functions differentiable in the variable $t$, twice differentiable in the variable $x$, possessing mixed second derivatives and bounded together with its derivatives on $\Omega_{T}$. Let $\bar{C}^{2}(\mathbb{R})$ be the space of the functions on $\mathbb{R}$ continuous and bounded with its derivatives up to the second order. By $C([0, T])$ we denote the space of the functions defined and continuous on the segment $[0, T]$.

We introduce conditions playing a key role in the proof of nonlocal solvability of Cauchy problem (4), (2):

$$
\begin{aligned}
& a_{1}(t)>0, \quad b_{1}(t)>0, \quad b_{2}(t)>0, \quad c_{1}(t)>0, \quad g_{1}(t)>0, \quad t \in[0, T], \\
& \varphi_{1}^{\prime}(x) \geqslant 0, \quad \varphi_{2}^{\prime}(x) \geqslant 0, \quad x \in \mathbb{R} .
\end{aligned}
$$

The next theorem provides conditions for the existence of local solution to Cauchy problem (4), (2), under which the solution $u(t, x)=w_{1}(t, t, x), v(t, x)=w_{2}(t, t, x)$ possesses the same smoothness in $x$ as the initial functions $\varphi_{1}(x), \varphi_{2}(x)$.

Theorem 1. Let $\varphi_{1}(x), \varphi_{2}(x) \in \bar{C}^{2}(\mathbb{R}), a_{1}(t), b_{1}(t), b_{2}(t), c_{1}(t), g_{1}(t) \in C([0, T])$ and conditions (28) be satisfied. Then for each $0 \leqslant t \leqslant T_{2}$, where $T_{2}=\min \left(\frac{1}{25 C_{\varphi} l}, \frac{1}{10 l}\right)$, Cauchy problem (4), (2) has the unique solution $u(t, x), v(t, x) \in \bar{C}^{1,2,2}\left(\Omega_{T_{2}}\right)$, which is determined by system of integral equations 24)-27).

The proof of the theorem is split into two lemmata. 
Lemma 2. Let $\varphi_{1}(x), \varphi_{2}(x) \in \bar{C}^{2}(\mathbb{R}), a_{1}(t), b_{1}(t), b_{2}(t), c_{1}(t), g_{1}(t) \in C([0, T])$ and conditions (28) hold. Then system of integral equations (24)-(27) possesses the unique solution $w_{j} \in \overline{C^{1,1}, 1}\left(\Gamma_{T_{2}}\right)$, where $j=\overline{1,4}$,

$$
T_{2}=\min \left(\frac{1}{25 C_{\varphi} l}, \frac{1}{10 l}\right) .
$$

Proof. The proof of the lemma follows the lines of [4] and this is why we mention only the milestones. The main difficulty is that system (24)-(27) involves the superposition of unknown functions. To overcome this problem, we employ a "two-level" algorithm of successive approximations.

The zero approximation for a solution to system of integral equations (24)-(27) is introduced by the identities:

$w_{10}(s, t, x)=\varphi_{1}(x), \quad w_{20}(s, t, x)=\varphi_{2}(x), \quad w_{30}(s, t, x)=\varphi_{2}(x), \quad w_{40}(s, t, x)=\varphi_{1}(x)$.

The first and next approximations for system of equations (24)-(27) are introduced by means of the recurrent sequence of equations $(n=1,2, \ldots)$ :

$$
\begin{aligned}
& w_{1 n}(s, t, x)=\varphi_{1}\left(x-\int_{0}^{t}\left(a_{1}(\tau) w_{1 n}+b_{1}(\tau) w_{3 n}\right) d \tau\right)+\int_{0}^{s}\left(a_{2} w_{1 n}+b_{2}(\tau) w_{3 n}\right) d \tau, \\
& w_{2 n}(s, t, x)=\varphi_{2}\left(x-\int_{0}^{t}\left(c_{1}(\tau) w_{4 n}(\tau, t, x)+g_{1}(\tau) w_{2 n}(\tau, t, x)\right) d \tau\right)+\int_{0}^{s} g_{2} w_{2 n}(\tau, t, x) d \tau, \\
& w_{3 n}(s, t, x)=w_{2(n-1)}\left(s, s, x-\int_{s}^{t}\left(a_{1}(\tau) w_{1 n}+b_{1}(\tau) w_{3 n}\right) d \tau\right), \\
& w_{4 n}(s, t, x)=w_{1(n-1)}\left(s, s, x-\int_{s}^{t}\left(c_{1}(\tau) w_{4 n}+g_{1}(\tau) w_{2 n}\right) d \tau\right) .
\end{aligned}
$$

For system of equations $(29)-32$, the zero approximation is defined by the identities

$$
w_{j n}^{0}=w_{j(n-1)}, j=\overline{1,4} .
$$

The first and next approximations are introduced by the relations:

$$
\begin{aligned}
& w_{1 n}^{k+1}(s, t, x)=\varphi_{1}\left(x-\int_{0}^{t}\left(a_{1}(\tau) w_{1 n}^{k}+b_{1}(\tau) w_{3 n}^{k}\right) d \tau\right)+\int_{0}^{s}\left(a_{2} w_{1 n}^{k}+b_{2}(\tau) w_{3 n}^{k}\right) d \tau, \\
& w_{2 n}^{k+1}(s, t, x)=\varphi_{2}\left(x-\int_{0}^{t}\left(c_{1}(\tau) w_{4 n}^{k}(\tau, t, x)+g_{1}(\tau) w_{2 n}^{k}(\tau, t, x)\right) d \tau\right)+\int_{0}^{s} g_{2} w_{2 n}^{k}(\tau, t, x) d \tau, \\
& w_{3 n}^{k+1}(s, t, x)=w_{2(n-1)}\left(s, s, x-\int_{s}^{t}\left(a_{1}(\tau) w_{1 n}^{k}+b_{1}(\tau) w_{3 n}^{k}\right) d \tau\right), \\
& w_{4 n}^{k+1}(s, t, x)=w_{1(n-1)}\left(s, s, x-\int_{s}^{t}\left(c_{1}(\tau) w_{4 n}^{k}+g_{1}(\tau) w_{2 n}^{k}\right) d \tau\right) .
\end{aligned}
$$

As in [2], [4], we establish that for all $0 \leqslant t \leqslant T_{1}$, where $T_{1}=\min \left(\frac{1}{20 C_{\varphi} l}, \frac{1}{4 l}\right)$, the successive approximations (33)-(36) converge to a continuous and bounded solution of system (29)-(32) 
and this solution possesses continuous bounded derivatives $\partial_{x} w_{j n}, j=\overline{1,4}$. The estimates hold:

$$
\begin{array}{ll}
\left\|w_{j n}\right\| \leqslant 2 C_{\varphi}, \quad j=\overline{1,4}, \quad & \left\|\partial_{x} w_{1 n}\right\| \leqslant 4 C_{\varphi}, \\
\left\|\partial_{x} w_{2 n}\right\| \leqslant 4 C_{\varphi}, & \left\|\partial_{x} w_{3 n}\right\| \leqslant 6 C_{\varphi}, \quad\left\|\partial_{x} w_{4 n}\right\| \leqslant 6 C_{\varphi} .
\end{array}
$$

Again as in [2], 4], we establish that for all $0 \leqslant t \leqslant T_{2}$, where $T_{2}=\min \left(\frac{1}{25 C_{\varphi} l}, \frac{1}{10 l}\right)$, successive approximations (29)-(32) converge to a continuous bonded solution of system (24)-(27), and this solution has continuous bounded derivatives $\partial_{x} w_{j}, j=\overline{1,4}$. The estimates hold true:

$$
\begin{aligned}
& \left\|w_{j}\right\| \leqslant 2 C_{\varphi}, \quad j=\overline{1,4}, \quad\left\|\partial_{x} w_{1}\right\| \leqslant 4 C_{\varphi}, \\
& \left\|\partial_{x} w_{2}\right\| \leqslant 4 C_{\varphi}, \quad \quad\left\|\partial_{x} w_{3}\right\| \leqslant 6 C_{\varphi}, \quad\left\|\partial_{x} w_{4}\right\| \leqslant 6 C_{\varphi} \text {. }
\end{aligned}
$$

In the same way as above we prove that $w_{j}, j=\overline{1,4}$ have continuous bounded derivatives with respect to the time $t$ on $\Gamma_{T_{2}}$. The uniqueness of the solution is proved as in paper [4].

Lemma 3. Let $\varphi_{1}(x), \varphi_{2}(x) \in \bar{C}^{2}(\mathbb{R}), a_{1}(t), b_{1}(t), b_{2}(t), c_{1}(t), g_{1}(t) \in C([0, T])$ and conditions (28) hold. Then the solutions $\left\{w_{j}\right\}, j=\overline{1,4}$ of the system of equations (24)-27) have continuous bounded derivatives $\frac{\partial^{2} w_{j}}{\partial x^{2}}, \frac{\partial^{2} w_{j}}{\partial x \partial t}, j=\overline{1,4}$ on $\Gamma_{T_{2}}$, where $T_{2}=\min \left(\frac{1}{25 C_{\varphi} l}, \frac{1}{10 l}\right)$.

Proof. We prove this lemma by the scheme proposed in [2] and we provide only the milestones. We twice differentiate successive approximations $29-(32)$ in $x$ and we denote $\omega_{j}^{n}=w_{j n x x}$, $j=\overline{1,4}$. As a result, we get the system of equations

$$
\begin{aligned}
\omega_{1}^{n}= & -\varphi_{1}^{\prime} \int_{0}^{t}\left(a_{1}(\tau) \omega_{1}^{n}+b_{1}(\tau) \omega_{3}^{n}\right) d \tau+\int_{0}^{s}\left(a_{2} \omega_{1}^{n}+b_{2}(\tau) \omega_{3}^{n}\right) d \tau \\
& +\varphi_{1}^{\prime \prime} \cdot\left(1-\int_{0}^{t}\left(a_{1}(\tau) w_{1 n x}+b_{1}(\tau) w_{3 n x}\right) d \tau\right)^{2}, \\
\omega_{2}^{n}= & -\varphi_{2}^{\prime} \int_{0}^{t}\left(c_{1}(\tau) \omega_{4}^{n}+g_{1}(\tau) \omega_{2}^{n}\right) d \tau+\int_{0}^{s} g_{2} \omega_{2}^{n} d \tau \\
& +\varphi_{2}^{\prime \prime} \cdot\left(1-\int_{0}^{t}\left(c_{1}(\tau) w_{4 n x}+g_{1}(\tau) w_{2 n x}\right) d \tau\right)^{2} \\
\omega_{3}^{n}= & \omega_{2}^{n-1} \cdot\left(1-\int_{s}^{t}\left(a_{1}(\tau) w_{1 n x}+b_{1}(\tau) w_{3 n x}\right) d \tau\right)^{2} \\
& -w_{2(n-1) x} \int_{s}^{t}\left(a_{1}(\tau) \omega_{1}^{n}+b_{1}(\tau) \omega_{3}^{n}\right) d \tau, \\
& -w_{1(n-1) x} \int_{s}^{t}\left(c_{1}(\tau) \omega_{4}^{n}+g_{1}(\tau) \omega_{2}^{n}\right) d \tau . \\
\omega_{4}^{n}= & \omega_{1}^{n-1} \cdot\left(1-\int_{s}^{t}\left(c_{1}(\tau) w_{4 n x}+g_{1}(\tau) w_{2 n x}\right) d \tau\right)^{2}
\end{aligned}
$$


Under conditions 280 , in view of the above estimates $\left\|w_{j n}\right\| \leqslant 2 C_{\varphi}, j=\overline{1,4}$, we obtain

$$
\begin{aligned}
& \left|\int_{s}^{t}\left(a_{1}(\tau) w_{1 n}+b_{1}(\tau) w_{3 n}\right) d \tau\right| \leqslant t l\left(\left\|w_{1 n}\right\|+\left\|w_{3 n}\right\|\right) \leqslant 4 t l C_{\varphi} \leqslant \frac{4 l C_{\varphi}}{25 l C_{\varphi}} \leqslant 0.16, \\
& \left|\int_{s}^{t}\left(c_{1}(\tau) w_{4 n}+g_{1}(\tau) w_{2 n}\right) d \tau\right| \leqslant t l\left(\left\|w_{4 n}\right\|+\left\|w_{2 n}\right\|\right) \leqslant 4 t l C_{\varphi} \leqslant \frac{4 l C_{\varphi}}{25 l C_{\varphi}} \leqslant 0.16 .
\end{aligned}
$$

We fix a point $x_{0} \in \mathbb{R}$ and we consider the set

$$
\Omega_{x_{0}}=\left\{x \mid x_{0}-0.16 \leqslant x \leqslant x_{0}+0.16\right\} .
$$

As in paper [2], under conditions (28), for all $x_{1}, x_{2} \in \mathbb{R}$, the inequalities hold:

$$
\begin{aligned}
& \left|\eta_{1 n}\left(s, t, x_{1}\right)-\eta_{1 n}\left(s, t, x_{2}\right)\right| \leqslant\left|x_{1}-x_{2}\right|, \\
& \left|\eta_{2 n}\left(s, t, x_{1}\right)-\eta_{2 n}\left(s, t, x_{2}\right)\right| \leqslant\left|x_{1}-x_{2}\right|,
\end{aligned}
$$

where

$$
\begin{aligned}
& \eta_{1 n}(s, t, x)=x-\int_{s}^{t}\left(a_{1}(\tau) w_{1 n}(\tau, t, x)+b_{1}(\tau) w_{3 n}(\tau, t, x)\right) d \tau, \\
& \eta_{2 n}(s, t, x)=x-\int_{s}^{t}\left(c_{1}(\tau) w_{4 n}(\tau, t, x)+g_{1}(\tau) w_{2 n}(\tau, t, x)\right) d \tau .
\end{aligned}
$$

In a closed bounded set $\Omega_{x_{0}}$, continuous second derivatives of the functions $\varphi_{i}, i=1$, are uniformly continuous. As in paper [2, we prove that under conditions (28), we have a uniform continuity of the functions $\omega_{1}^{n}, \omega_{2}^{n}$ in $x \in \Omega_{x_{0}}$, which implies the equicontinuity of the functions $\omega_{1}^{n}, \omega_{2}^{n}$ in $x$ at an arbitrary point $x_{0} \in \mathbb{R}$. The equicontinuity of the functions $\omega_{1}^{n}, \omega_{2}^{n}$ in $x$ is employed for proving the convergence of successive approximations $\omega_{j}^{n}, j=\overline{1,4}$.

We consider the system of equations

$$
\begin{aligned}
\tilde{\omega}_{1}^{n}= & -\varphi_{1}^{\prime}\left(\eta_{1}(0, t, x)\right) \int_{0}^{t}\left(a_{1}(\tau) \tilde{\omega}_{1}^{n}+b_{1}(\tau) \tilde{\omega}_{3}^{n}\right) d \tau+\int_{0}^{s}\left(a_{2} \tilde{\omega}_{1}^{n}+b_{2}(\tau) \tilde{\omega}_{3}^{n}\right) d \tau \\
& +\varphi_{1}^{\prime \prime} \cdot\left(1-\int_{0}^{t}\left(a_{1}(\tau) w_{1 x}+b_{1}(\tau) w_{3 x}\right) d \tau\right)^{2}, \\
\tilde{\omega}_{2}^{n}= & -\varphi_{2}^{\prime}\left(\eta_{2}(0, t, x)\right) \int_{0}^{t}\left(c_{1}(\tau) \tilde{\omega}_{4}^{n}+g_{1}(\tau) \tilde{\omega}_{2}^{n}\right) d \tau+\int_{0}^{s} g_{2} \tilde{\omega}_{2}^{n} d \tau \\
& +\varphi_{2}^{\prime \prime} \cdot\left(1-\int_{0}^{t}\left(c_{1}(\tau) w_{4 x}+g_{1}(\tau) w_{2 x}\right) d \tau\right)^{2}, \\
\tilde{\omega}_{3}^{n}= & \tilde{\omega}_{2}^{n-1} \cdot\left(1-\int_{s}^{t}\left(a_{1}(\tau) w_{1 x}+b_{1}(\tau) w_{3 x}\right) d \tau\right)^{2}-w_{2 x}\left(s, s, \eta_{1}(s, t, x)\right) \int_{s}^{t}\left(a_{1}(\tau) \tilde{\omega}_{1}^{n}+b_{1}(\tau) \tilde{\omega}_{3}^{n}\right) d \tau \\
& \left(1-\int_{s}^{t}\left(c_{1}(\tau) w_{4 x}+g_{1}(\tau) w_{2 x}\right) d \tau\right)^{2}-w_{1 x}\left(s, s, \eta_{2}(s, t, x)\right) \int_{s}\left(c_{1}(\tau) \tilde{\omega}_{4}^{n}+g_{1}(\tau) \tilde{\omega}_{2}^{n}\right) d \tau .
\end{aligned}
$$


Under conditions (28), on $\Gamma_{T_{2}}$ we have $\tilde{\omega}_{j}^{n} \rightarrow \tilde{\omega}_{j}, j=\overline{1,4}$, and the estimates hold:

$$
\left\|\tilde{\omega}_{1}\right\| \leqslant 2 C_{\varphi}, \quad\left\|\tilde{\omega}_{2}\right\| \leqslant 2 C_{\varphi}, \quad\left\|\tilde{\omega}_{3}\right\| \leqslant 3 C_{\varphi}, \quad\left\|\tilde{\omega}_{4}\right\| \leqslant 3 C_{\varphi} .
$$

Then we establish that under conditions (28), on $\Gamma_{T_{2}}$, the successive approximations $\omega_{j}^{n}$ converge to the functions $\tilde{\omega}_{j}, j=\overline{1,4}$, as $n \rightarrow \infty$.

We obtain that under conditions (28), $w_{j n x x} \rightarrow w_{j x x}=\tilde{\omega}_{j}$, where the functions $\frac{\partial^{2} w_{j}}{\partial x^{2}}, j=\overline{1,4}$, are continuous and bounded on $\Gamma_{T_{2}}$. Then we establish that under conditions (28) there exist continuous and bounded derivatives $\frac{\partial^{2} w_{j}}{\partial x \partial t}, j=\overline{1,4}$, on $\Gamma_{T_{2}}$.

\section{EXISTENCE OF NONLOCAL SOLUTION}

There holds the following theorem on sufficient conditions ensuring the existence and uniqueness of the solution to the Cauchy problem in the original coordinates for a given finite segment $t \in[0, T]$.

Theorem 2. Let $\varphi_{1}(x), \varphi_{2}(x) \in \bar{C}^{2}(\mathbb{R}), a_{1}(t), b_{1}(t), b_{2}(t), c_{1}(t), g_{1}(t) \in C([0, T])$ and conditions (28) hold. Then for each $T>0$, Cauchy problem (4), (2) has the unique solution $u(t, x), v(t, x) \in \bar{C}^{1,2,2}\left(\Omega_{T}\right)$, which is determined by the system of integral equations (24)-27).

Proof. We differentiate the system of equations (4) in $x$ and denoting $p(t, x)=u_{x}(t, x), q(t, x)=$ $v_{x}(t, x)$, we obtain the system of equations:

$$
\left\{\begin{array}{l}
\partial_{t} p+\left(a_{1}(t) u(t, x)+b_{1}(t) v(t, x)\right) \partial_{x} p=-a_{1}(t) p^{2}-b_{1}(t) p q+a_{2} p+b_{2}(t) q, \\
\partial_{t} q+\left(c_{1}(t) u(t, x)+g_{1}(t) v(t, x)\right) \partial_{x} q=-g_{1}(t) q^{2}-c_{1}(t) p q+g_{2} q \\
p(0, x)=\varphi_{1}^{\prime}(x), \quad q(0, x)=\varphi_{2}^{\prime}(x) .
\end{array}\right.
$$

We add two equations to the system of equations $(18)-(23)$ :

$$
\left\{\begin{array}{l}
\frac{d \gamma_{1}(s, t, x)}{d s}=-a_{1}(s) \gamma_{1}^{2}-b_{1}(s) \gamma_{1} \gamma_{2}\left(s, s, \eta_{1}\right)+a_{2} \gamma_{1}+b_{2}(s) \gamma_{2}\left(s, s, \eta_{1}\right), \\
\frac{d \gamma_{2}(s, t, x)}{d s}=-g_{1}(s) \gamma_{2}^{2}-c_{1}(s) \gamma_{1}\left(s, s, \eta_{2}\right) \gamma_{2}+g_{2} \gamma_{2}
\end{array}\right.
$$

subject to the conditions $\gamma_{1}(0, t, x)=\varphi_{1}^{\prime}\left(\eta_{1}\right), \gamma_{2}(0, t, x)=\varphi_{2}^{\prime}\left(\eta_{2}\right)$.

We rewrite system of equations (44) as follows:

$$
\left\{\begin{array}{l}
\gamma_{1}(s, t, x)=\varphi_{1}^{\prime}\left(\eta_{1}\right)+\int_{0}^{s}\left[-a_{1}(\tau) \gamma_{1}^{2}+\left(b_{2}(\tau)-b_{1}(\tau) \gamma_{1}\right) \gamma_{2}\left(\tau, \tau, \eta_{1}\right)+a_{2} \gamma_{1}\right] d \tau \\
\gamma_{2}(s, t, x)=\varphi_{2}^{\prime}\left(\eta_{2}\right)+\int_{0}^{s}\left[-g_{1}(\tau) \gamma_{2}^{2}-c_{1}(\tau) \gamma_{1}\left(\tau, \tau, \eta_{2}\right) \gamma_{2}+g_{2} \gamma_{2}\right] d \tau
\end{array}\right.
$$

The existence of a continuous solution to system (45) on $\Gamma_{T_{2}}$, where $T_{2}=\min \left(\frac{1}{25 C_{\varphi} l}, \frac{1}{10 l}\right)$, under conditions (28), is proved by the method of successive approximations. We define these approximations:

$$
\left\{\begin{array}{l}
\gamma_{1}^{n+1}=\varphi_{1}^{\prime}\left(\eta_{1}\right)+\int_{0}^{s}\left[-a_{1}(\tau)\left(\gamma_{1}^{n}\right)^{2}+\left(b_{2}(\tau)-b_{1}(\tau) \gamma_{1}^{n}\right) \gamma_{2}^{n}\left(\tau, \tau, \eta_{1}\right)+a_{2} \gamma_{1}^{n}\right] d \tau \\
\gamma_{2}^{n+1}=\varphi_{2}^{\prime}\left(\eta_{2}\right)+\int_{0}^{s}\left[-g_{1}(\tau)\left(\gamma_{2}^{n}\right)^{2}-c_{1}(\tau) \gamma_{1}^{n}\left(\tau, \tau, \eta_{2}\right) \gamma_{2}^{n}+g_{2} \gamma_{2}^{n}\right] d \tau
\end{array}\right.
$$


and also $\gamma_{1}^{0}=\varphi_{1}^{\prime}\left(\eta_{1}\right), \gamma_{2}^{0}=\varphi_{2}^{\prime}\left(\eta_{2}\right)$. Under conditions $(28)$, on $\Gamma_{T_{2}}$ the estimates hold: $\left|\gamma_{i}^{n+1}\right| \leqslant$ $2 C_{\varphi}$.

As in paper [2], we establish that for all $0 \leqslant t \leqslant T_{2}$, where $T_{2}=\min \left(\frac{1}{25 C_{\varphi} l}, \frac{1}{10 l}\right)$, the inequality

$$
\left\|\gamma_{1}^{n+1}-\gamma_{1}^{n}\right\|+\left\|\gamma_{2}^{n+1}-\gamma_{2}^{n}\right\| \leqslant 0.52\left(\left\|\gamma_{1}^{n}-\gamma_{1}^{n-1}\right\|+\left\|\gamma_{2}^{n}-\gamma_{2}^{n-1}\right\|\right),
$$

holds true. This inequality implies that the successive approximations $\left\{\gamma_{i}^{n}\right\}, i=1,2$, converge to a continuous solution of system (45) on $\Gamma_{T_{2}}$ under conditions (28). The solution satisfies the estimates:

$$
\left|\gamma_{i}\right| \leqslant 2 C_{\varphi}, \quad i=1,2
$$

We differentiate successive approximations (46) with respect to $x$ :

$$
\left\{\begin{aligned}
\gamma_{1 x}^{n+1}= & \varphi_{1}^{\prime \prime}\left(\eta_{1}\right) \eta_{1 x} \\
& +\int_{0}^{s}\left(\left(a_{2}-2 a_{1}(\tau) \gamma_{1}^{n}-b_{1}(\tau) \gamma_{2}^{n}\right) \gamma_{1 x}^{n}+\left(b_{2}(\tau)-b_{1}(\tau) \gamma_{1}^{n}\right) \gamma_{2 x}^{n}\left(\tau, \tau, \eta_{1}\right) \eta_{1 x}\right) d \tau \\
\gamma_{2 x}^{n+1}= & \varphi_{2}^{\prime \prime}\left(\eta_{2}\right) \eta_{2 x}+\int_{0}^{s}\left[\left(g_{2}-2 g_{1}(\tau) \gamma_{2}^{n}-c_{1}(\tau) \gamma_{1}^{n}\right) \gamma_{2 x}^{n}-c_{1}(\tau) \gamma_{2}^{n} \gamma_{1 x}^{n}\left(\tau, \tau, \eta_{2}\right) \eta_{2 x}\right] d \tau,
\end{aligned}\right.
$$

where

$$
\begin{aligned}
& \eta_{1 x}(s, t, x)=1-\int_{s}^{t}\left(a_{1}(\tau) w_{1 x}+b_{1}(\tau) w_{3 x}\right) d \tau, \\
& \eta_{2 x}(s, t, x)=1-\int_{s}^{t}\left(c_{1}(\tau) w_{4 x}+g_{1}(\tau) w_{2 x}\right) d \tau .
\end{aligned}
$$

Under conditions 28$)$, on $\Gamma_{T_{2}}$, the estimates

$$
\left|\eta_{i x}\right| \leqslant 1, \quad\left|\gamma_{i x}^{n+1}\right| \leqslant 5 C_{\varphi}, \quad i=1,2,
$$

hold.

We consider the system of equations:

$$
\left\{\begin{aligned}
\omega_{21}= & \varphi_{1}^{\prime \prime}\left(\eta_{1}\right) \eta_{1 x} \\
& +\int_{0}^{s}\left(\left(a_{2}-2 a_{1}(\tau) \gamma_{1}-b_{1}(\tau) \gamma_{2}\right) \omega_{21}+\left(b_{2}(\tau)-b_{1}(\tau) \gamma_{1}\right) \omega_{22}\left(\tau, \tau, \eta_{1}\right) \eta_{1 x}\right) d \tau, \\
\omega_{22}= & \varphi_{2}^{\prime \prime}\left(\eta_{2}\right) \eta_{2 x}+\int_{0}^{s}\left(\left(g_{2}-2 g_{1}(\tau) \gamma_{2}-c_{1}(\tau) \gamma_{1}\right) \omega_{22}-c_{1}(\tau) \omega_{21}\left(\tau, \tau, \eta_{2}\right) \gamma_{2} \eta_{2 x}\right) d \tau .
\end{aligned}\right.
$$

We prove the existence of a continuous solution to system (48) by means of the method of successive approximations:

$$
\left\{\begin{aligned}
\omega_{21}^{n+1}= & \varphi_{1}^{\prime \prime}\left(\eta_{1}\right) \eta_{1 x} \\
& +\int_{0}^{s}\left[\left(a_{2}-2 a_{1}(\tau) \gamma_{1}-b_{1}(\tau) \gamma_{2}\right) \omega_{21}^{n}+\left(b_{2}(\tau)-b_{1}(\tau) \gamma_{1}\right) \omega_{22}^{n}\left(\tau, \tau, \eta_{1}\right) \eta_{1 x}\right] d \tau \\
\omega_{22}^{n+1}= & \varphi_{2}^{\prime \prime}\left(\eta_{2}\right) \eta_{2 x}+\int_{0}^{s}\left[\left(g_{2}-2 g_{1}(\tau) \gamma_{2}-c_{1}(\tau) \gamma_{1}\right) \omega_{22}^{n}-c_{1}(\tau) \omega_{21}^{n}\left(\tau, \tau, \eta_{2}\right) \gamma_{2} \eta_{2 x}\right] d \tau
\end{aligned}\right.
$$


Under conditions (28), on $\Gamma_{T_{2}}$, the estimates

$$
\left\|\omega_{2 i}^{n+1}\right\| \leqslant 5 C_{\varphi}, \quad i=1,2,
$$

hold true.

It follows from (49) that

$$
\begin{aligned}
\left|\omega_{21}^{n+1}-\omega_{21}^{n}\right| \leqslant & \left|\int_{0}^{s}\left(a_{2}-2 a_{1}(\tau) \gamma_{1}-b_{1}(\tau) \gamma_{2}\right)\left(\omega_{21}^{n}-\omega_{21}^{n-1}\right) d \tau\right| \\
& +\left|\int_{0}^{s}\left(b_{2}(\tau)-b_{1}(\tau) \gamma_{1}\right)\left(\omega_{22}^{n}\left(\tau, \tau, \eta_{1}\right)-\omega_{22}^{n-1}\left(\tau, \tau, \eta_{1}\right)\right) \eta_{1 x} d \tau\right| \\
\left|\omega_{22}^{n+1}-\omega_{22}^{n}\right| \leqslant & \left|\int_{0}^{s}\left(g_{2}-2 g_{1}(\tau) \gamma_{2}-c_{1}(\tau) \gamma_{1}\right)\left(\omega_{22}^{n}-\omega_{22}^{n-1}\right) d \tau\right| \\
& +\left|\int_{0}^{s} c_{1}(\tau) \gamma_{2}\left(\omega_{21}^{n}\left(\tau, \tau, \eta_{2}\right)-\omega_{21}^{n-1}\left(\tau, \tau, \eta_{2}\right)\right) \eta_{2 x} d \tau\right|
\end{aligned}
$$

By the properties of integrals, absolute value, supremum of the functions, we arrive at the following inequalities:

$$
\begin{aligned}
\left|\omega_{21}^{n+1}-\omega_{21}^{n}\right| \leqslant & l \int_{0}^{s}\left(1+2\left|\gamma_{1}\right|+\left|\gamma_{2}\right|\right)\left|\omega_{21}^{n}-\omega_{21}^{n-1}\right| d \tau \\
& +l \int_{0}^{s}\left(1+\left|\gamma_{1}\right|\right) \cdot\left|\omega_{22}^{n}\left(\tau, \tau, \eta_{1}\right)-\omega_{22}^{n-1}\left(\tau, \tau, \eta_{1}\right)\right| \cdot\left|\eta_{1 x}\right| d \tau \\
\left|\omega_{22}^{n+1}-\omega_{22}^{n}\right| \leqslant & l \int_{0}^{s}\left(1+2\left|\gamma_{2}\right|+\left|\gamma_{1}\right|\right)\left|\omega_{22}^{n}-\omega_{22}^{n-1}\right| d \tau \\
& +l \int_{0}^{s}\left|\gamma_{2}\right| \cdot\left|\omega_{21}^{n}\left(\tau, \tau, \eta_{2}\right)-\omega_{21}^{n-1}\left(\tau, \tau, \eta_{2}\right)\right| \cdot\left|\eta_{2 x}\right| d \tau .
\end{aligned}
$$

Since

$$
\left|\gamma_{i}\right| \leqslant 2 C_{\varphi}, \quad i=1,2, \quad\left|\eta_{1 x}\right| \leqslant 1
$$

we have

$$
\left\|\omega_{21}^{n+1}-\omega_{21}^{n}\right\| \leqslant\left(6 l t C_{\varphi}+l t\right)\left\|\omega_{21}^{n}-\omega_{21}^{n-1}\right\|+\left(2 l t C_{\varphi}+l t\right)\left\|\omega_{22}^{n}-\omega_{22}^{n-1}\right\| .
$$

Since

$$
\left|\gamma_{i}\right| \leqslant 2 C_{\varphi}, \quad i=1,2, \quad\left|\eta_{2 x}\right| \leqslant 1
$$

we get

$$
\left\|\omega_{22}^{n+1}-\omega_{22}^{n}\right\| \leqslant\left(6 l t C_{\varphi}+l t\right)\left\|\omega_{22}^{n}-\omega_{22}^{n-1}\right\|+2 l t C_{\varphi}\left\|\omega_{21}^{n}-\omega_{21}^{n-1}\right\| .
$$

We sum up two latter inequalities to obtain

$$
\left\|\omega_{21}^{n+1}-\omega_{21}^{n}\right\|+\left\|\omega_{22}^{n+1}-\omega_{22}^{n}\right\| \leqslant\left(8 l t C_{\varphi}+l t\right)\left\|\omega_{21}^{n}-\omega_{21}^{n-1}\right\|+\left(8 l t C_{\varphi}+2 l t\right)\left\|\omega_{22}^{n}-\omega_{22}^{n-1}\right\| .
$$

For all $0 \leqslant t \leqslant T_{2}$ with $T_{2}=\min \left(\frac{1}{25 C_{\varphi} l}, \frac{1}{10 l}\right)$, the inequality holds:

$$
\left\|\omega_{21}^{n+1}-\omega_{21}^{n}\right\|+\left\|\omega_{22}^{n+1}-\omega_{22}^{n}\right\| \leqslant\left(\frac{8 l C_{\varphi}}{25 C_{\varphi} l}+\frac{l}{10 l}\right)\left\|\omega_{21}^{n}-\omega_{21}^{n-1}\right\|
$$




$$
\begin{gathered}
+\left(\frac{8 l C_{\varphi}}{25 C_{\varphi} l}+\frac{2 l}{10 l}\right)\left\|\omega_{22}^{n}-\omega_{22}^{n-1}\right\|, \\
\left\|\omega_{21}^{n+1}-\omega_{21}^{n}\right\|+\left\|\omega_{22}^{n+1}-\omega_{22}^{n}\right\| \leqslant 0.42\left\|\omega_{21}^{n}-\omega_{21}^{n-1}\right\|+0.52\left\|\omega_{22}^{n}-\omega_{22}^{n-1}\right\|, \\
\left\|\omega_{21}^{n+1}-\omega_{21}^{n}\right\|+\left\|\omega_{22}^{n+1}-\omega_{22}^{n}\right\| \leqslant 0.52\left(\left\|\omega_{21}^{n}-\omega_{21}^{n-1}\right\|+\left\|\omega_{22}^{n}-\omega_{22}^{n-1}\right\|\right) .
\end{gathered}
$$

Hence, under conditions (28), successive approximations $\left\{\omega_{2 i}^{n}\right\}, i=1,2$, converge to a continuous solution of system (48) on $\Gamma_{T_{2}}$. It follows from (47), (48) that

$$
\begin{aligned}
\left\|\gamma_{1 x}^{n+1}-\omega_{21}\right\| \leqslant & \left|\int_{0}^{s}\left(a_{2}-2 a_{1}(\tau) \gamma_{1}-b_{1}(\tau) \gamma_{2}\left(\tau, \tau, \eta_{1}\right)\right)\left(\gamma_{1 x}^{n}-\omega_{21}\right) d \tau\right| \\
& +\left|\int_{0}^{s}\left(b_{2}(\tau)-b_{1}(\tau) \gamma_{1}\right)\left(\gamma_{2 x}^{n}\left(\tau, \tau, \eta_{1}\right)-\omega_{22}\left(\tau, \tau, \eta_{1}\right)\right) \eta_{1 x} d \tau\right|+\left|\sigma_{31}^{n}\right|, \\
\left\|\gamma_{2 x}^{n+1}-\omega_{22}\right\| \leqslant & \left|\int_{0}^{s}\left(g_{2}-2 g_{1}(\tau) \gamma_{2}-c_{1}(\tau) \gamma_{1}\left(\tau, \tau, \eta_{2}\right)\right)\left(\gamma_{2 x}^{n}-\omega_{22}\right) d \tau\right| \\
& +\left|\int_{0}^{s}\left(-c_{1}(\tau) \gamma_{2}\right)\left(\gamma_{1 x}^{n}\left(\tau, \tau, \eta_{2}\right)-\omega_{21}\left(\tau, \tau, \eta_{2}\right)\right) \eta_{2 x} d \tau\right|+\left|\sigma_{41}^{n}\right|,
\end{aligned}
$$

where

$$
\begin{aligned}
\sigma_{31}^{n} & =\int_{0}^{s}\left[\left(-2 a_{1}(\tau) \gamma_{1 x}^{n}-b_{1}(\tau) \gamma_{2 x}^{n} \eta_{1 x}\right)\left(\gamma_{1}^{n}-\gamma_{1}\right)-b_{1}(\tau) \gamma_{1 x}^{n}\left(\gamma_{2}^{n}-\gamma_{2}\right)\right] d \tau \\
\sigma_{41}^{n} & =\int_{0}^{s}\left[\left(-2 g_{1}(\tau) \gamma_{2 x}^{n}-c_{1}(\tau) \gamma_{1 x}^{n} \eta_{2 x}\right)\left(\gamma_{2}^{n}-\gamma_{2}\right)-c_{1}(\tau) \gamma_{2 x}^{n}\left(\gamma_{1}^{n}-\gamma_{1}\right)\right] d \tau
\end{aligned}
$$

By the properties of integrals, absolute value and supremum of function we obtain the following inequalities:

$$
\begin{aligned}
\left|\gamma_{1 x}^{n+1}-\omega_{21}\right| \leqslant & l \int_{0}^{s}\left(1+2\left|\gamma_{1}\right|+\left|\gamma_{2}\right|\right)\left|\gamma_{1 x}^{n}-\omega_{21}\right| d \tau \\
& +l \int_{0}^{s}\left(1+\left|\gamma_{1}\right|\right) \cdot\left|\gamma_{2 x}^{n}\left(\tau, \tau, \eta_{1}\right)-\omega_{22}\left(\tau, \tau, \eta_{1}\right)\right| \cdot\left|\eta_{1 x}\right| d \tau+\left|\sigma_{31}^{n}\right| \\
\left|\gamma_{2 x}^{n+1}-\omega_{22}\right| \leqslant & l \int_{0}^{s}\left(1+2\left|\gamma_{2}\right|+\left|\gamma_{1}\right|\right)\left|\gamma_{2 x}^{n}-\omega_{22}\right| d \tau \\
& +l \int_{0}^{s}\left|\gamma_{2}\right| \cdot\left|\gamma_{1 x}^{n}\left(\tau, \tau, \eta_{2}\right)-\omega_{21}\left(\tau, \tau, \eta_{2}\right)\right| \cdot\left|\eta_{2 x}\right| d \tau+\left|\sigma_{41}^{n}\right|
\end{aligned}
$$

Since

we have

$$
\left|\gamma_{1}\right| \leqslant 2 C_{\varphi},\left|\gamma_{2}\right| \leqslant 2 C_{\varphi}, \quad\left|\eta_{1 x}\right| \leqslant 1, \quad\left|\eta_{2 x}\right| \leqslant 1
$$

$$
\begin{aligned}
& \left\|\gamma_{1 x}^{n+1}-\omega_{21}\right\| \leqslant\left(6 l t C_{\varphi}+l t\right)\left\|\gamma_{1 x}^{n}-\omega_{21}\right\|+\left(2 l t C_{\varphi}+l t\right)\left\|\gamma_{2 x}^{n}-\omega_{22}\right\|+\left|\sigma_{31}^{n}\right| \\
& \left\|\gamma_{2 x}^{n+1}-\omega_{22}\right\| \leqslant\left(6 l t C_{\varphi}+l t\right)\left\|\gamma_{2 x}^{n}-\omega_{22}\right\|+2 l t C_{\varphi}\left\|\gamma_{1 x}^{n}-\omega_{21}\right\|+\left|\sigma_{41}^{n}\right|
\end{aligned}
$$


We sum up to latter inequalities and obtain:

$$
\begin{aligned}
\left\|\gamma_{1 x}^{n+1}-\omega_{21}\right\|+\left\|\gamma_{2 x}^{n+1}-\omega_{22}\right\| \leqslant & \left(8 l t C_{\varphi}+l t\right)\left\|\gamma_{1 x}^{n}-\omega_{21}\right\| \\
& +\left(8 l t C_{\varphi}+2 l t\right)\left\|\gamma_{2 x}^{n}-\omega_{22}\right\|+\left|\sigma_{31}^{n}\right|+\left|\sigma_{41}^{n}\right| .
\end{aligned}
$$

For all $0 \leqslant t \leqslant T_{2}$, where $T_{2}=\min \left(\frac{1}{25 C_{\varphi} l}, \frac{1}{10 l}\right)$, the inequality holds true:

$$
\begin{aligned}
& \left\|\gamma_{1 x}^{n+1}-\omega_{21}\right\|+\left\|\gamma_{2 x}^{n+1}-\omega_{22}\right\| \leqslant 0.42\left\|\gamma_{1 x}^{n}-\omega_{21}\right\|+0.52\left\|\gamma_{2 x}^{n}-\omega_{22}\right\|+\left|\sigma_{31}^{n}\right|+\left|\sigma_{41}^{n}\right|, \\
& \left\|\gamma_{1 x}^{n+1}-\omega_{21}\right\|+\left\|\gamma_{2 x}^{n+1}-\omega_{22}\right\| \leqslant 0.52\left(\left\|\gamma_{1 x}^{n}-\omega_{21}\right\|+\left\|\gamma_{2 x}^{n}-\omega_{22}\right\|\right)+\left|\sigma_{31}^{n}\right|+\left|\sigma_{41}^{n}\right| .
\end{aligned}
$$

Employing the uniform convergences $\gamma_{1}^{n} \Rightarrow \gamma_{1}, \gamma_{2}^{n} \Rightarrow \gamma_{2}$, we choose $n=N$ so that $\left|\sigma_{31}^{n}\right|+\left|\sigma_{41}^{n}\right|<$ $\varepsilon$. Then

$$
\left\|\gamma_{1 x}^{n+1}-\omega_{21}\right\|+\left\|\gamma_{2 x}^{n+1}-\omega_{22}\right\| \leqslant 0.52\left(\left\|\gamma_{1 x}^{n}-\omega_{21}\right\|+\left\|\gamma_{2 x}^{n}-\omega_{22}\right\|\right)+\varepsilon
$$

We denote $S_{1 N}=\left\|\gamma_{1 x}^{N}-\omega_{21}\right\|+\left\|\gamma_{2 x}^{N}-\omega_{22}\right\|$.

By means of the induction, we are going to prove the inequality:

$$
\left\|\gamma_{1 x}^{N+p}-\omega_{21}\right\|+\left\|\gamma_{2 x}^{N+p}-\omega_{22}\right\| \leqslant(0.52)^{p} S_{1 N}+\left(1+0.52+\cdots+(0.52)^{p-1}\right) \varepsilon,
$$

where $p=1,2, \ldots$ It follows from that for $N+1$, the inequality

$$
\left\|\gamma_{1 x}^{N+1}-\omega_{21}\right\|+\left\|\gamma_{2 x}^{N+1}-\omega_{22}\right\| \leqslant 0.52 S_{1 N}+\varepsilon
$$

holds as well as

$$
\begin{aligned}
& \left\|\gamma_{1 x}^{N+2}-\omega_{21}\right\|+\left\|\gamma_{2 x}^{N+2}-\omega_{22}\right\| \leqslant 0.52\left(\left\|\gamma_{1 x}^{N+1}-\omega_{21}\right\|+\left\|\gamma_{2 x}^{N+1}-\omega_{22}\right\|\right), \\
& \left\|\gamma_{1 x}^{N+2}-\omega_{21}\right\|+\left\|\gamma_{2 x}^{N+2}-\omega_{22}\right\| \leqslant 0.52\left(0.52 S_{1 N}+\varepsilon\right)+\varepsilon
\end{aligned}
$$

Hence, for $N+2$ we get:

$$
\left\|\gamma_{1 x}^{N+2}-\omega_{21}\right\|+\left\|\gamma_{2 x}^{N+2}-\omega_{22}\right\| \leqslant(0.52)^{2} S_{1 N}+(1+0.52) \varepsilon
$$

Suppose that for $N+p, p=1,2, \ldots$, the inequality holds:

$$
\left\|\gamma_{1 x}^{N+p}-\omega_{21}\right\|+\left\|\gamma_{2 x}^{N+p}-\omega_{22}\right\| \leqslant(0.52)^{p} S_{1 N}+\left(1+0.52+\cdots+(0.52)^{p-1}\right) \varepsilon .
$$

Then for $N+p+1, p=1,2, \ldots$ we infer that

$$
\begin{aligned}
& \left\|\gamma_{1 x}^{N+p+1}-\omega_{21}\right\|+\left\|\gamma_{2 x}^{N+p+1}-\omega_{22}\right\| \leqslant 0.52\left(\left\|\gamma_{1 x}^{N+p}-\omega_{21}\right\|+\left\|\gamma_{2 x}^{N+p}-\omega_{22}\right\|\right)+\varepsilon, \\
& \left\|\gamma_{1 x}^{N+p+1}-\omega_{21}\right\|+\left\|\gamma_{2 x}^{N+p+1}-\omega_{22}\right\| \leqslant 0.52\left((0.52)^{p} S_{1 N}+\left(1+0.52+\cdots+(0.52)^{p-1}\right) \varepsilon\right)+\varepsilon, \\
& \left\|\gamma_{1 x}^{N+p+1}-\omega_{21}\right\|+\left\|\gamma_{2 x}^{N+p+1}-\omega_{22}\right\| \leqslant(0.52)^{p+1} S_{1 N}+\left(1+0.52+\cdots+(0.52)^{p}\right) \varepsilon .
\end{aligned}
$$

Therefore, inequality (51) is true, where $p=1,2, \ldots$ and this implies that

$$
\begin{aligned}
& \left\|\gamma_{1 x}^{N+p}-\omega_{21}\right\|+\left\|\gamma_{2 x}^{N+p}-\omega_{22}\right\|<(0.52)^{p} S_{1 N}+\frac{1}{1-0.52} \varepsilon, \\
& \left\|\gamma_{1 x}^{N+p}-\omega_{21}\right\|+\left\|\gamma_{2 x}^{N+p}-\omega_{22}\right\|<(0.52)^{p} S_{1 N}+\frac{1}{0.48} \varepsilon .
\end{aligned}
$$

Therefore,

$$
\left\|\gamma_{1 x}^{N+p}-\omega_{21}\right\|+\left\|\gamma_{2 x}^{N+p}-\omega_{22}\right\| \rightarrow 0 \quad \text { as } \quad N \rightarrow \infty, \quad p \rightarrow \infty .
$$

Hence, $\lim _{n \rightarrow \infty} \gamma_{i x}^{n}=\omega_{2 i}, i=1,2$. We obtain that the solution to system 45 possesses a continuous derivative in $x: \gamma_{i x}=\frac{\partial \gamma_{i}}{\partial x}=\omega_{2 i}$. The estimates hold:

$$
\left\|\gamma_{i x}\right\| \leqslant 5 C_{\varphi}, \quad i=1,2 .
$$


In the same way as in paper [1, we prove that the solution of system 45) possesses a continuous derivative in $t$. We first differentiate system of equations 45 with respect to $t$ :

$$
\left\{\begin{aligned}
\gamma_{1 t}(s, t, x)= & \varphi_{1}^{\prime \prime}\left(\eta_{1}\right) \eta_{1 t} \\
& +\int_{0}^{s}\left[\left(a_{2}-2 a_{1}(\tau) \gamma_{1}-b_{1}(\tau) \gamma_{2}\left(\tau, \tau, \eta_{1}\right)\right) \gamma_{1 t}+\left(b_{2}(\tau)-b_{1}(\tau) \gamma_{1}\right) \gamma_{2 x} \eta_{1 t}\right] d \tau \\
\gamma_{2 t}(s, t, x)= & \varphi_{2}^{\prime \prime}\left(\eta_{2}\right) \eta_{2 t} \\
& +\int_{0}^{s}\left[\left(g_{2}-2 g_{1}(\tau) \gamma_{2}-c_{1}(\tau) \gamma_{1}\left(\tau, \tau, \eta_{2}\right)\right) \gamma_{2 t}-c_{1}(\tau) \gamma_{2} \gamma_{1 x} \eta_{2 t}\right] d \tau
\end{aligned}\right.
$$

where

$$
\begin{aligned}
& \eta_{1 t}(s, t, x)=-a_{1}(t) w_{1}-b_{1}(t) w_{3}-\int_{s}^{t}\left(a_{1}(\tau) w_{1 t}+b_{1}(\tau) w_{3 t}\right) d \tau, \\
& \eta_{2 t}(s, t, x)=-c_{1}(t) w_{4}-g_{1}(t) w_{2}-\int_{s}^{t}\left(c_{1}(\tau) w_{4 t}+g_{1}(\tau) w_{2 t}\right) d \tau .
\end{aligned}
$$

We differentiate system of equations (44) with respect to $t$ :

$$
\left\{\begin{array}{l}
\frac{d \gamma_{1 t}(s, t, x)}{d s}=\left(a_{2}-2 a_{1}(s) \gamma_{1}-b_{1}(s) \gamma_{2}\left(s, s, \eta_{1}\right)\right) \gamma_{1 t}+\left(b_{2}(s)-b_{1}(s) \gamma_{1}\right) \gamma_{2 x} \eta_{1 t}, \\
\frac{d \gamma_{2 t}(s, t, x)}{d s}=\left(g_{2}-2 g_{1}(s) \gamma_{2}-c_{1}(s) \gamma_{1}\left(s, s, \eta_{2}\right)\right) \gamma_{2 t}-c_{1}(s) \gamma_{1 x} \gamma_{2} \eta_{2 t}
\end{array}\right.
$$

subject to the conditions: $\gamma_{1 t}(0, t, x)=\varphi_{1}^{\prime \prime}\left(\eta_{1}\right) \eta_{1 t}, \gamma_{2 t}(0, t, x)=\varphi_{2}^{\prime \prime}\left(\eta_{2}\right) \eta_{2 t}$. Therefore,

$$
\begin{aligned}
\gamma_{1 t}= & \varphi_{1}^{\prime \prime}\left(\eta_{1}\right) \eta_{1 t} \exp \left(\int_{0}^{s}\left(a_{2}-2 a_{1}(\tau) \gamma_{1}-b_{1}(\tau) \gamma_{2}\left(\tau, \tau, \eta_{1}\right)\right) d \tau\right) \\
& +\int_{0}^{s}\left(b_{2}(\tau)-b_{1}(\tau) \gamma_{1}\right) \gamma_{2 x} \eta_{1 t} \exp \left(\int_{\tau}^{s}\left(a_{2}-2 a_{1}(\nu) \gamma_{1}-b_{1}(\nu) \gamma_{2}\left(\nu, \nu, \eta_{1}\right)\right) d \nu\right) d \tau \\
\gamma_{2 t}= & \varphi_{2}^{\prime \prime}\left(\eta_{2}\right) \eta_{2 t} \exp \left(\int_{0}^{s}\left(g_{2}-2 g_{1}(\tau) \gamma_{2}-c_{1}(\tau) \gamma_{1}\left(\tau, \tau, \eta_{2}\right)\right) d \tau\right) \\
& -\int_{0}^{s} c_{1}(\tau) \gamma_{2} \gamma_{1 x} \eta_{2 t} \exp \left(\int_{\tau}^{s}\left(g_{2}-2 g_{1}(\nu) \gamma_{2}-c_{1}(\nu) \gamma_{1}\left(\nu, \nu, \eta_{2}\right)\right) d \nu\right) d \tau
\end{aligned}
$$

Therefore, there exits functions $\gamma_{1 t}, \gamma_{2 t}$ satisfying system (52). Then we differentiate with respect to $t$ successive approximations (46) and under conditions (28), on $\Gamma_{T_{2}}$ we prove the convergence $\gamma_{1 t}^{n} \rightarrow \gamma_{1 t}, \gamma_{2 t}^{n} \rightarrow \gamma_{2 t}$. Hence, we establish that

$$
\gamma_{1 t}=\frac{\partial \gamma_{1}}{\partial t}, \quad \gamma_{2 t}=\frac{\partial \gamma_{2}}{\partial t}
$$

Thus, we have proved the existence of a differentiable solution to problem (45). Therefore,

$$
\gamma_{1}(t, t, x)=p(t, x)=\partial_{x} u, \quad \gamma_{2}(t, t, x)=q(t, x)=\partial_{x} v .
$$

As in paper [2], we establish estimates:

$$
\|v\| \leqslant C_{\varphi} \exp \left(\left|g_{2}\right| T\right), \quad\|u\| \leqslant C_{\varphi} \exp \left(\left|a_{2}\right| T\right)\left(1+T l \exp \left(\left|g_{2}\right| T\right)\right) .
$$


By (44) we have

$$
\left\{\begin{aligned}
\gamma_{1}(s, t, x)= & \varphi_{1}^{\prime}\left(\eta_{1}\right) \exp \left(-\int_{0}^{s}\left(a_{1}(\tau) \gamma_{1}+b_{1}(\tau) \gamma_{2}\left(\tau, \tau, \eta_{1}\right)-a_{2}\right) d \tau\right) \\
& +\int_{0}^{s} b_{2}(\tau) \gamma_{2}\left(\tau, \tau, \eta_{1}\right) \exp \left(-\int_{\tau}^{s}\left(a_{1}(\tau) \gamma_{1}+b_{1}(\tau) \gamma_{2}\left(\nu, \nu, \eta_{1}\right)-a_{2}\right) d \nu\right) d \tau, \\
\gamma_{2}(s, t, x)= & \varphi_{2}^{\prime}\left(\eta_{2}\right) \exp \left(-\int_{0}^{s}\left(g_{1}(\tau) \gamma_{2}+c_{1}(\tau) \gamma_{1}\left(\tau, \tau, \eta_{2}\right)-g_{2}\right) d \tau\right) .
\end{aligned}\right.
$$

Since $\varphi_{2}^{\prime}(x) \geqslant 0, x \in \mathbb{R}$, it follows from t55) that $\gamma_{2} \geqslant 0$ on $\Gamma_{T}$. And due to

$$
\varphi_{1}^{\prime}(x) \geqslant 0, \quad x \in \mathbb{R}, \quad b_{2}(t)>0, \quad \gamma_{2} \geqslant 0 \quad \text { on } \Gamma_{T},
$$

it follows from 55 that $\gamma_{1} \geqslant 0$ on $\Gamma_{T}$. Since

$$
\begin{array}{ll}
\gamma_{1} \geqslant 0, & \gamma_{2} \geqslant 0 \quad \text { on } \quad \Gamma_{T}, \\
a_{1}(t)>0, & b_{1}(t)>0, \quad b_{2}(t)>0, \quad c_{1}(t)>0, \quad g_{1}(t)>0, \quad t \in[0, T],
\end{array}
$$

by (55) we get that on $\Gamma_{T}$ the estimates hold:

$$
\left\|\gamma_{2}\right\| \leqslant C_{\varphi} \exp \left(\left|g_{2}\right| T\right), \quad\left\|\gamma_{1}\right\| \leqslant C_{\varphi} \exp \left(\left|a_{2}\right| T\right)\left(1+T l \exp \left(\left|g_{2}\right| T\right)\right)
$$

Hence,

$$
\left\|\partial_{x} v\right\| \leqslant C_{\varphi} \exp \left(\left|g_{2}\right| T\right), \quad\left\|\partial_{x} u\right\| \leqslant C_{\varphi} \exp \left(\left|a_{2}\right| T\right)\left(1+T l \exp \left(\left|g_{2}\right| T\right)\right) .
$$

As in [1], we confirm that for all $t$ and $x$ the estimates

$$
\begin{aligned}
& \left|\partial_{x^{2}}^{2} u\right| \leqslant E_{11} \operatorname{ch}\left(T \sqrt{C_{12} C_{21}}\right)+E_{21} \sqrt{\frac{C_{12}}{C_{21}}} \operatorname{sh}\left(T \sqrt{C_{12} C_{21}}\right), \\
& \left|\partial_{x^{2}}^{2} v\right| \leqslant E_{21} \operatorname{ch}\left(T \sqrt{C_{12} C_{21}}\right)+E_{11} \sqrt{\frac{C_{21}}{C_{12}}} \operatorname{sh}\left(T \sqrt{C_{12} C_{21}}\right),
\end{aligned}
$$

hold, where $E_{11}, E_{21}, C_{12}, C_{21}$ are constants determined by initial data.

The obtained global estimates (54), (56)-(58) allow us to continue the solution to each prescribed segment $[0, T]$. We choose $u\left(T_{0}, x\right), v\left(T_{0}, x\right)$ as the initial data and employing Theorem 1, we continue the solution to some segment $\left[T_{0}, T_{1}\right]$. Then we take $u\left(T_{1}, x\right), v\left(T_{1}, x\right)$ and employing Theorem 1, we continue the solution to the segment $\left[T_{1}, T_{2}\right]$. In finitely many steps the solution can be continued to each prescribed segment $[0, T]$.

The uniqueness of solution to Cauchy problem (4), (2) can be proved by applying estimates similar to those employed in proving the convergence of successive approximations.

Example. We consider the Cauchy problem for the system

$$
\left\{\begin{array}{l}
\partial_{t} u(t, x)+((t+1) u(t, x)+(2 t+1) v(t, x)) \partial_{x} u(t, x)=-3 u(t, x)+(2 t+3) v(t, x), \\
\partial_{t} v(t, x)+((t+2) u(t, x)+(2 t+4) v(t, x)) \partial_{x} v(t, x)=-2 v(t, x),
\end{array}\right.
$$

where $u(t, x), v(t, x)$ are unknown functions, $(t, x) \in \Omega_{T}$, subject to the initial conditions:

$$
u(0, x)=\varphi_{1}(x)=1+5 \arctan x, \quad v(0, x)=\varphi_{2}(x)=-\frac{1}{e^{x}+2} .
$$

Since $\varphi_{1}(x), \varphi_{2}(x) \in \bar{C}^{2}(\mathbb{R}), a_{1}(t), b_{1}(t), b_{2}(t), c_{1}(t), g_{1}(t) \in C([0, T])$,

$$
\begin{aligned}
& a_{1}(t)=t+1>0, \quad b_{1}(t)=2 t+1>0, \quad b_{2}(t)=2 t+3>0, \quad c_{1}(t)=t+2>0, \\
& g_{1}(t)=2 t+4>0, \quad t \in[0, T], \quad \varphi_{1}^{\prime}(x)=\frac{5}{1+x^{2}}>0, \quad \varphi_{2}^{\prime}(x)=\frac{e^{x}}{\left(e^{x}+2\right)^{2}}>0, \quad x \in \mathbb{R},
\end{aligned}
$$


then by Theorem 2, Cauchy problem (59), (60) possesses the unique solution $u(t, x), v(t, x) \in$ $\bar{C}^{1,2,2}\left(\Omega_{T}\right)$.

\section{BIBLIOGRAPHY}

1. S.N. Alekseenko, T.A. Shemyakina, M.V. Dontsova. Nonlocal solvability conditions for system of first order partial differential equations // Nauchno-tekhnicheskie vedomosti SPbGBU. Fiz.-Mat. Nauki. 3(177). 190-201 (2013). (in Russian).

2. M.V. Dontsova. Nonlocal solvability conditions for Cauchy problem for a system of first order partial differential equations with special right-hand sides // Ufimskij Matem. Zhurn. 6:4, 71-82 (2014). [Ufa Math. J. 6:4, 68-80 (2014).]

3. B.L. Rozhdestvenskij, N.N. Yanenko. Systems of quasilinear equations and their applications to gas dynamics. Nauka, Moscow (1978). [Amer. Math. Soc., Providence, RI (1983).]

4. M.I. Imanaliev, S.N. Alekseenko. On the existence of a smooth bounded solution for a system of two first-order nonlinear partial differential equations // Dokl. Ross. Akad. Nauk. 379:1, 16-21 (2001). [Dokl. Math. 64:1, 10-15 (2001).]

5. M.I. Imanaliev, P.S. Pankov, S.N. Alekseenko. Additional argument method // Vestnik KazNU. Ser. Matem. mech. inform. 1, 60-64 (2006). (in Russian).

6. M.V. Dontsova. Study of solvability for a system of first order partial differential equations with free terms // Proc. of International Youth Conference "Lomonosov-2014", (2014). (in Russian).

7. T.A. Shemyakina. Existence theorem for bounded solution to Cauchy problem for Frankl system of hyperbolic type // Nauchno-tekhnicheskie vedomosti SPbGBU. Fiz.-Mat. Nauki. 2(146), 130-131 (2012). (in Russian).

8. S.N. Alekseenko, M.V. Dontsova. The solvability conditions of the system of long waves in a water rectangular channel, the depth of which varies along the axis // Zhurn. Srednevolzh. Matem. Obsch. 18:2, 115-124 (2016). (in Russian).

9. M.V. Dontsova. Nonlocale existence of a bounded solution to a system of two first order partial differential equations with continuous bounded right hand sides // Vestnik TvGU. Ser. Prikl. Matem. 3, 21-36 (2014). (in Russian).

Marina Vladimirovna Dontsova,

Lobachevsky University,

Gagarin av. 23,

603950, Nizhny Novgorod, Russia

E-mail: dontsowa.marina2011@yandex.ru 